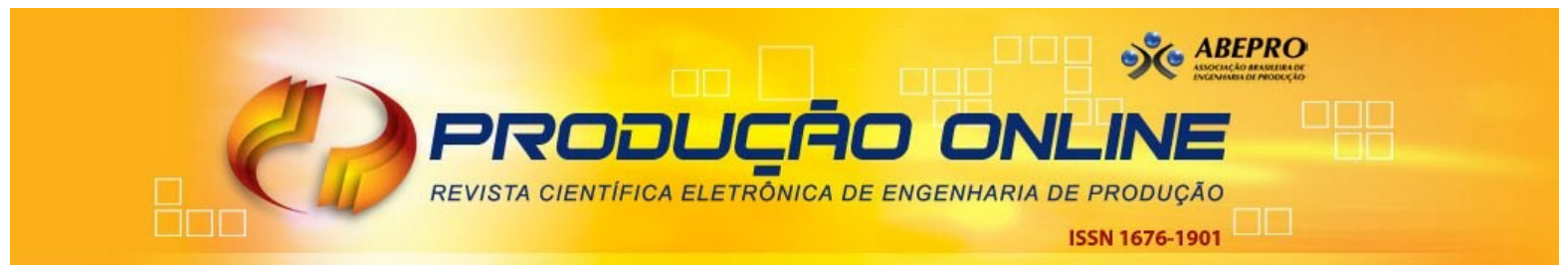

\title{
ESTRATÉGIA DE MANUTENÇÃO BASEADA EM FUNÇÕES DE CONFIABILIDADE PARA UMA BOMBA CENTRÍFUGA PETROLÍFERA
}

\section{MAINTENANCE STRATEGY BASED ON RELIABILITY FUNCTIONS FOR AN OIL CENTRIFUGAL PUMP}

\author{
Denis Carlos Mengue* E-mail: denismengue@bol.com.br \\ Miguel Afonso Sellitto*E-mail: sellitto@unisinos.br \\ *Universidade do Vale do Rio dos Sinos(UNISINOS), São Leopoldo, RS
}

\begin{abstract}
Resumo: O objetivo deste artigo foi definir a estratégia de manutenção mais adequada para uma bomba centrífuga (preventiva, preditiva, corretiva ou emergencial), com base em cálculos de confiabilidade. O método de pesquisa foi a modelagem quantitativa, aplicada em uma planta petrolífera. $\mathrm{O}$ estudo pode contribuir para o aprimoramento de um modelo estratégico para a gestão da manutenção industrial da planta. Foram levantados, no sistema de informações da empresa, registros de onze anos dos tempos entre falhas e dos tempos para reparo do equipamento. Estes tempos foram modelados por distribuições de probabilidade. A partir dos resultados obtidos, foram calculadas as funções Confiabilidade $R(t)$ e Mantenabilidade $M(t)$. Pela combinação de seus valores esperados (MTBF e MTTR), foi calculada a disponibilidade Av da bomba. Os valores para MTBF, MTTR e Av foram respectivamente 3.936 horas, 133 horas e $96,73 \%$. O fator de forma mais provável da distribuição de Weibull que modelou o tempo até a falha foi de 0,69 . Com isso, foi possível afirmar que a bomba está em fase de mortalidade infantil. Pelo referencial teórico da RCM, a estratégia de manutenção indicada é a corretiva. Esta estratégia visa eliminar os defeitos de projeto do equipamento, reforçar os itens que quebraram e remover as causas de origem das falhas.
\end{abstract}

Palavras-chave: RCM. Confiabilidade. Mantenabilidade. Análise de Weibull. Manutenção.

Abstract: The purpose of this article was to define the most appropriate maintenance strategy for a centrifugal pump (preventive, predictive, corrective or emergency), based on reliability calculation. The research method was the quantitative modeling, applied in a petroleum plant. The study may contribute to the development of a strategic model for the management of the industrial plant maintenance. In the company's information system, records of eleven years have been gathered on times between failures and times for repair of the equipment. These times were modeled by probability distributions. From the results obtained were calculated Reliability and Maintainability functions $R(t)$ and $\mathrm{M}(\mathrm{t})$. By combining their expected values (MTBF and MTTR), Av availability was calculated. The figures for MTBF, MTTR and Av were respectively 3,936 hours, 133 hours and $96.73 \%$. The most likely shape factor of the Weibull distribution which modeled the time between failures was 0.69 . So, it was possible to affirm that the pump is in the infant mortality phase. The theoretical framework of RCM indicated corrective maintenance as the strategy for the item. This strategy aims to eliminate the defects of equipment design, reinforce items that usually break and remove the causes of the failures.

Key-words: RCM. Reliability. Maintainability. Weibull Analysis. Maintenance. 


\section{INTRODUÇÃO}

No passado, a manutenção industrial foi considerada como fator gerador de custos, muitas vezes desnecessários. Mais recentemente, a função manutenção passou a ser mais considerada no cenário de negócios industriais, pois a estratégia de manutenção do parque fabril pode influenciar positiva ou negativamente prioridades competitivas da manufatura, tais como custo, qualidade e flexibilidade (PINJALA et al., 2006). Com a automação em larga escala e a redução de estoques exigida pelos sistemas de produção Just-in-Time, a manutenção aumentou de importância na estratégia de produção: rupturas de fluxos produtivos podem rapidamente causar dano econômico à manufatura (MÁRQUEZ e GUPTA, 2006).

A importância crescente da manufatura na estratégia empresarial tem exigido mais articulação e estratégia na execução da função manutenção, devido principalmente à maior necessidade de disponibilidade de máquinas ao longo do tempo (RAMOS FILHO et al., 2010). Como tais ativos demandam elevados investimentos, sua utilização deve ser maximizada para maximizar retornos (RAPOSO, 2011). Segundo Márquez et al. (2009), equipamentos operando fora de suas condições ótimas podem gerar perdas irrecuperáveis em mercados competitivos. Muitas vezes, custos relacionados a perdas em processos são decorrentes de falhas no planejamento ou na execução da manutenção industrial. Tais falhas repercutem na confiabilidade do processo produtivo, afetando a competitividade do negócio e da empresa na indústria (PARDIA e CHATTOPADHYAY, 2007). Portanto, em contexto de competitividade, as empresas devem garantir confiabilidade e disponibilidade dos equipamentos por métodos de gestão estratégica da manutenção (TSANG, 2002; RAPOSO, 2011).

A especificação de estratégias de manutenção nos diferentes setores da indústria ou da prestação de serviços pode ser orientada pelo uso de técnicas e métodos quantitativos. Várias técnicas e métodos baseados em abordagens quantitativas foram estudados e aplicados à manutenção nos últimos anos. A RCM (Reliability-Centered Maintenance - Manutenção Centrada na Confiabilidade) é um destes métodos (SELLITTO, 2007). A RCM tem como metas: a identificação dos modos de falha e suas consequências; a escolha da técnica de manutenção de melhor custo-benefício; e o acompanhamento da aplicação a fim de minimizar o 
risco e o impacto da falha. Garante-se a funcionalidade do equipamento com confiabilidade e disponibilidade a baixo custo e com segurança (MACCHI et al., 2012). A RCM é adequada para empresas de processamento contínuo, tais como refinarias de petróleo. Neste tipo de empresa, as corridas de produção são longas e o tamanho do lote é grande. O produto é sempre o mesmo, com eventuais variações de matérias-primas e o impacto do resultado da manutenção no resultado do processo usualmente é grande (WUTKE e SELLITTO, 2008).

O objetivo deste artigo é definir a estratégia de manutenção para uma bomba centrífuga instalada em uma planta petrolífera. A bomba está instalada em um sistema tecnológico complexo, sujeito ao desgaste e a intervenções incompletas de manutenção. O método de pesquisa foi a modelagem quantitativa. Definido o ponto do ciclo de vida em que o equipamento se encontra, foi proposta uma estratégia de manutenção. Também é objetivo do artigo contribuir para o aprimoramento de um modelo estratégico para a gestão da manutenção de plantas petrolíferas.

A literatura traz relatos de aplicação de técnicas semelhantes às usadas neste artigo. Dentre muitos outros estudos, alguns podem ser citados, pois auxiliaram na pesquisa. Mahmood et al. (2011) relacionaram estratégia de manutenção com estratégia de produção em plantas petrolíferas. Mendes (2011) utilizou simulação de Monte Carlo e análise de sensibilidade para dar suporte à elaboração de planos de manutenção para dois cenários de produção distintos, um Just-in-time e outro protegido por estoques. Guzzon (2009) calculou o nível de confiabilidade global dos sistemas de controle e de propulsão de um sistema automatizado de transporte de passageiros por meio da transformação da FMEA (Failure mode and effect analisys - Análise do efeito de modos de falha) em parâmetros para as distribuições das probabilidades dos componentes envolvidos. Salgado (2008) usou técnicas de inteligência computacional, por dados quantitativos para modelagem e análise de confiabilidade e mantenabilidade para sistemas reparáveis e não reparáveis, visando à solução de problemas de otimização de confiabilidade e definição de arquitetura e componentes do sistema. Lopes e Samohyl (2003) usaram Análise de Componentes Principais para determinação do tempo médio entre falhas e da confiabilidade dos equipamentos por combinação linear de variáveis originais, para a obtenção de novas variáveis de análise, os componentes principais. Neste caso, a função de confiabilidade é entendida como a confiabilidade da componente principal gerada 
em função da variabilidade do tempo de falha das peças que compõem o equipamento. Sellitto (2007 e 2005) usou funções de confiabilidade e modelos matemáticos para a definição de estratégias de manutenção e intervalos entre intervenções preventivas em equipamentos industriais. Wuttke e Sellitto (2008) calcularam a disponibilidade de uma válvula de processo petroquímico. Brand (2011) calculou a confiabilidade sistêmica de uma linha de produção da indústria metalmecânica e em seguida estipulou estratégias de manutenção individuais para os equipamentos da linha. Lucatelli (2002) e Raposo (2004) aplicaram funções de confiabilidade respectivamente em manutenção de equipamentos hospitalares e em distribuição de energia elétrica. Santos (2003) usou métodos estatísticos para o cálculo de intervalos de manutenção preventiva em equipamentos industriais.

O restante do artigo está organizado em: revisão bibliográfica; descrição da pesquisa; discussão dos resultados; e considerações finais.

\section{CONFIABILIDADE, MANUTENIBILIDADE E DISPONIBILIDADE EM MANUTENÇÃO}

Sistemas de produção têm incorporado novas funcionalidades que aumentaram a complexidade de operações e equipamentos e têm exigido mais confiabilidade, mantenabilidade e segurança dos processos produtivos. Com isso, técnicas de engenharia mais avançadas têm sido usadas para garantir o projeto, operação e manutenção dos sistemas produtivos com custos adequados, riscos controlados e desempenho esperado (RAPOSO, 2011).

O uso da confiabilidade em Manutenção originou-se a partir de análises de falhas em equipamentos eletrônicos de uso militar durante a década de 1950, nos Estados Unidos. Em 1960 foi criado um grupo para o aperfeiçoamento de um programa específico de confiabilidade dentro da Federal Aviation Administration FAA (SELLITTO, 2005). Sendo um processo estocástico, as incertezas ligadas aos parâmetros das funções devem ser consideradas. São necessárias massas de dados com tamanho e acuracidade suficientes (NIKOLOPOULOS et al., 2003).

A confiabilidade de um equipamento pode ser definida como a probabilidade que desempenhe sua função durante um período estabelecido, sob determinadas condições de uso (ELSAYED, 1996). Segundo Fritsch e Ribeiro (1998), a Revista Produção Online, Florianópolis, SC, v.13, n. 2, p. 759-783, abr./jun. 2013. 
confiabilidade não é medida somente pela conformidade técnica e pela qualidade do projeto, mas também pela boa impressão dos clientes em relação ao fabricante pela comercialização de produtos confiáveis ao longo do tempo. Portanto, o estudo de confiabilidade é um ciclo que começa nas fases de pesquisa, projeto e fabricação e se consolida durante a utilização dos produtos pelos clientes. Para Guzzon (2009), a análise de confiabilidade vai desde o início do projeto até as fases de desenvolvimento e operação. Seu objetivo é reduzir a probabilidade de falhas a que o sistema ou equipamento está sujeito ou ainda minimizar o efeito negativo das mesmas, utilizando-se dados quantitativos oferecidos pelo processo.

Lafraia (2001) relata que a confiabilidade está ligada à confiança que se tenha em um produto, equipamento ou sistema, esperando que o mesmo não venha a falhar. $\mathrm{O}$ autor cita que a confiabilidade deve ser tratada na fase de projeto, por meio do tratamento probabilístico das variáveis aleatórias que estão envolvidas no mesmo. Após entrega e instalação do equipamento, pouca coisa adicional pode ser feita para alterar a confiabilidade, apenas para mantê-la em patamares aceitáveis.

A confiabilidade em um dado momento é representada por uma probabilidade. O modelo matemático mais usual para representar a condição de um item é o binário: um equipamento está operacional ou está em falha, não havendo estágio intermediário (FOGLIATTO e RIBEIRO, 2009).

\subsection{Modelos de funções de confiabilidade}

A determinação da função confiabilidade é possível pela modelagem, por uma distribuição de probabilidade, dos tempos até a falha do objeto de estudo. A modelagem oferece outras informações importantes, tais como a probabilidade de sobrevivência até o tempo $t$, o tempo médio até a falha e a função risco $h(t)$ do equipamento (FOGLIATTO e RIBEIRO, 2009). Segundo Lewis (1996), distribuições de probabilidade são necessárias para modelar o tempo até a falha, pois não se consegue conhecer e controlar todos os fatores ativos que o afeta.

Fogliatto e Ribeiro (2009) apontam as distribuições exponencial, Weibull, gamma e log-normal como as mais comumente empregadas para descrever os tempos até a falha de equipamentos. Citam também a distribuição normal, porém consideram que a mesma é menos aplicada em estudos de confiabilidade. Ireson et 
al. (1996) citam três funções e um parâmetro como importantes em estudos de confiabilidade: a função densidade de probabilidade de falha $f(t)$, a função confiabilidade $R(t)$, a função de risco $h(t)$, e o tempo médio até a falha MTTF.

As distribuições gamma e de Weibull apresentam peculiaridades que interessam à gestão da manutenção.

A distribuição gamma descreve sistemas que operam em paralelo. O tempo para a falha de um sistema que opera com componentes em paralelo, constituído por $n$ componentes independentes, tal como bombas ou compressores, é o tempo total até que o último componente venha a falhar e segue uma distribuição gamma (DODSON e NOLAN, 2002).

A distribuição de Weibull é importante devido à sua flexibilidade, pois pode modelar comportamentos diferentes para a função de risco $h(t)$ (risco crescente, decrescente ou constante). $O$ valor de seu parâmetro de forma $\gamma$ define se o risco de falha do equipamento é crescente, decrescente ou constante. Se $\gamma<1, h(t)$ é decrescente; se $\gamma=1, h(t)$ é constante; e se $\gamma>1, h(t)$ é crescente. Outra característica relevante da distribuição de Weibull é que ela modela falhas de equipamentos sujeitos a $n$ modos de falha em série: a falha ocorre quando o primeiro de $n$ modos de falha, que competem entre si, se materializa. Por fim, outras distribuições podem ser entendidas como casos particulares da distribuição de Weibull. Se $\gamma=1$, Weibull recai na exponencial; se $\gamma=2$, Weibull recai na distribuição de Rayleigh; se $\gamma=2,5$, Weibull tem comportamento semelhante à lognormal; se $\gamma=$ 3,2, Weibull tem comportamento semelhante à normal (LEWIS, 1996; KAPUR e LAMBERSON, 1977).

O modelo de confiabilidade baseado na distribuição de Weibull segue as expressões dadas pelas equações de (1) a (4) (FOGLIATTO e RIBEIRO, 2009).

$$
\begin{gathered}
f(t)=\frac{\gamma}{\theta}\left(\frac{t-t_{0}}{\theta}\right)^{\gamma-1} e\left[-\left(\frac{t-t_{0}}{\theta}\right)^{\gamma}\right] \quad 0 \leq t \leq \infty \\
h(t)=\frac{\gamma}{\theta}\left(\frac{t-t_{0}}{\theta}\right)^{\gamma-1} \\
R(t)=e^{-\left(\frac{t-t_{0}}{\theta}\right)^{\gamma}}
\end{gathered}
$$

Revista Produção Online, Florianópolis, SC, v.13, n. 2, p. 759-783, abr./jun. 2013. 


$$
M T B F=t_{0}+\theta^{1 / \gamma} \Gamma\left(1+\frac{1}{\gamma}\right)
$$

Em que:

$t_{0}=$ tempo isento de falha;

$\gamma$ = parâmetro de forma;

$\theta$ = parâmetro de escala;

$t=$ tempo até a falha.

As expressões matemáticas de outras distribuições e comentários sobre os comportamentos que estas modelam são encontrados em Fogliatto e Ribeiro (2009) e em Elsayed (1996). Mais comentários sobre modelagens de sistemas em engenharia e outras distribuições são encontrados em Shapiro e Hahn (1967).

\subsection{Manutenibilidade e disponibilidade}

A função manutenibilidade ou mantenabilidade está ligada ao esforço despendido na atividade de manutenção. O tempo médio de manutenção compreende o tempo para isolamento da falha, para o reparo e para os testes necessários para que a produção volte ao seu ritmo normal. Apesar de a eficiência da gestão de manutenção influenciar no tempo total gasto no reparo, a maior parte deste tempo é devida a decisões que foram tomadas na fase de projeto do equipamento (LAFRAIA, 2001).

A Norma NBR ISO 14224 conceitua a mantenabilidade como a probabilidade de que um item possa ser restaurado de volta a uma dada condição, dentro de um período de tempo determinado, quando a manutenção é efetuada por profissional que possui níveis de habilidade específicos e usando procedimentos e recursos prescritos (NBR ISO 14224, 2011, p.182).

As distribuições que mais usualmente descrevem a função mantenabilidade são a normal e a lognormal. Sellitto (2005) afirma que a normal se ajusta a casos em que a tarefa é composta por uma sequência serial de $n$ atividades. Pelo teorema do limite central, quando $n$ tende a infinito, a soma das $n$ atividades tende a uma normal. Esta situação ocorre na desmontagem e remontagem de máquinas e na 
construção de infra-estrutura para a atividade. O autor também afirma que a lognormal se aplica quando a atividade requer mais estratégia e habilidades cognitivas, citando como justificativa a lei de Fletcher-Weber para atividades que envolvam inteligência.

Para este artigo interessa mais a lognormal.

Se uma variável aleatória $x$ é definida como $x=\ln t$, então $x$ é normalmente distribuída com uma média de $\mu$ e desvio padrão de $\sigma$. A função densidade de probabilidade da lognormal é mostrada em (5), onde $t$ representa o tempo até o reparo, com média do logaritmo dos dados $\mu$ e desvio padrão do logaritmo dos dados $\sigma$ (KAPUR e LAMBERSON, 1977).

$$
f(t)=\frac{1}{\sigma t \sqrt{2} \pi} e^{\left[-\frac{1}{2} \ln \left(\frac{\mu-t}{\sigma}\right)^{2}\right]}
$$

A disponibilidade $A v(t)$ é a probabilidade de que um equipamento esteja disponível no momento em que for requisitado para operar. A disponibilidade aumenta quando aumenta o intervalo entre falhas e aumenta quando diminui o tempo até o reparo. É possível demonstrar que a disponibilidade $A v(t)$ depende do tempo médio entre falhas e do tempo médio até o reparo (RAUSAND e HOYLAND, 2004). Esta dependência é representada pela equação 6.

$$
A v(t)=\frac{M T B F}{M T B F+M T T R}
$$

\section{ESTRATÉGIAS DE MANUTENÇÃO BASEADAS NA FUNÇÃO RISCO}

A análise de Weibull pode ser entendida como um método estatístico que modela dados de falhas a uma distribuição específica de Weibull. Uma das possibilidades oferecidas pela análise é identificar se a falha é um evento prematuro, randômico ou ocasionada por desgaste, segundo o formato da função risco $h(t)$. Função risco $h(t)$ decrescente aponta para eventos prematuros; $h(t)$ constante aponta para eventos randômicos; e $h(t)$ decrescente aponta para falhas por 
desgates. A viabilização deste tipo de análise depende de um histórico confiável de dados de intervenções de manutenção (GROSH, 1989).

Um dos modos de visualizar a análise pela $h(t)$ é usando a chamada curva da banheira, uma construção abstrata que apresenta as três fases de vida de componentes individuais. A cada período podem ser associados diferentes tipos de falha. Nem todos os tipos de equipamentos ou sistemas mostram todas as fases da curva como, por exemplo, um programa de computador que se enquadra como um sistema que possui somente a fase de mortalidade infantil (LAFRAIA, 2001). Sellitto (2005) associa a cada faixa do fator de forma uma fase da curva da banheira e uma estratégia de manutenção. A Figura 1 sintetiza a relação entre a curva da banheira e o fator de forma.

Figura 1 - Curva da banheira e ciclo de vida de equipamentos (Fonte: Sellitto, 2005)

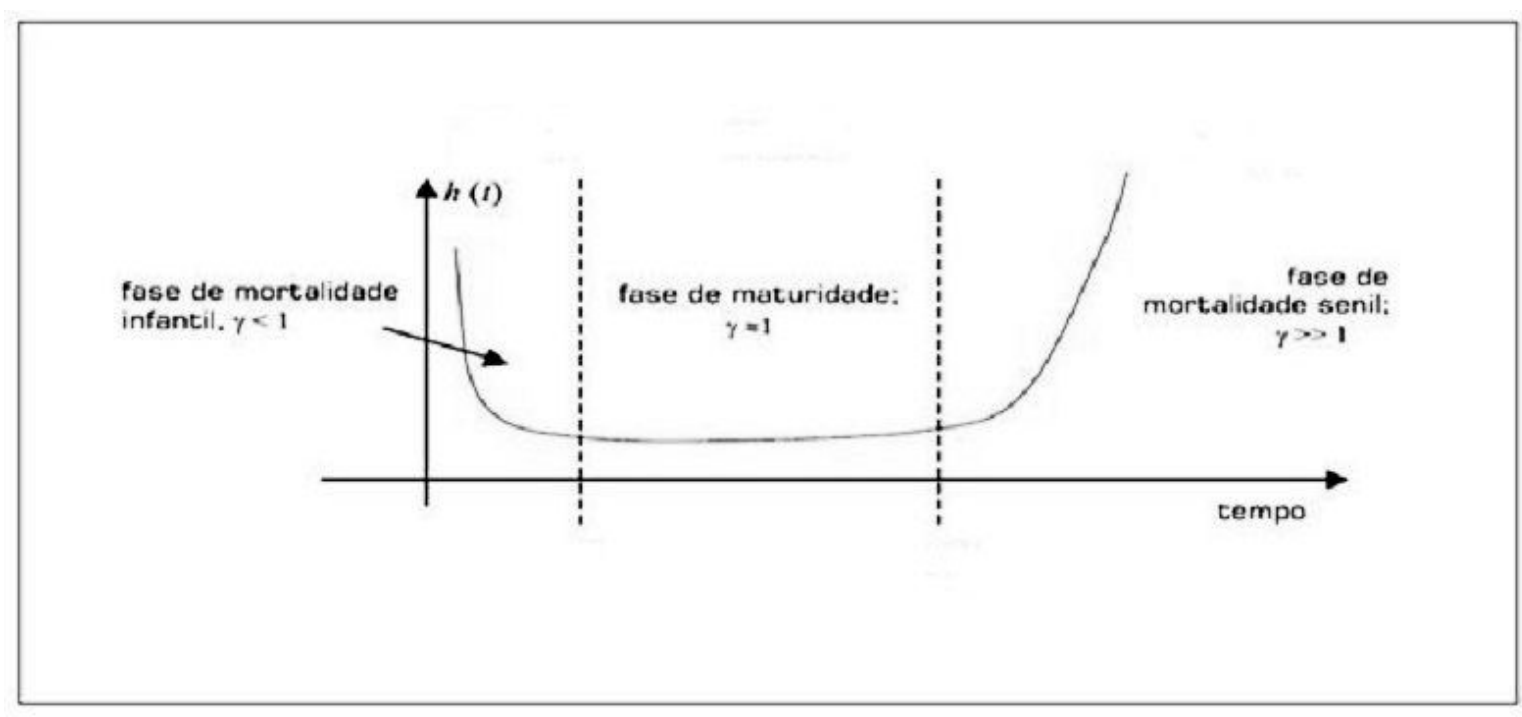

O primeiro período é a mortalidade infantil. Nesta região ocorrem falhas prematuras, tais como erros nos processos de fabricação, na instalação ou no uso de materiais. Uma estratégia para o período é a manutenção corretiva, na qual se descobrem e se eliminam as deficiências de projeto. A prática continuada da manutenção corretiva ou engenharia de manutenção permite que o equipamento evolua para a maturidade (MACCHI et al., 2012).

O período subsequente é a maturidade. Nesta, o equipamento apresenta taxa de falhas constante, causada por falhas casuais, tais como fator de segurança insuficiente ou erros humanos durante a operação. Sellitto (2005) relaciona a esta

Revista Produção Online, Florianópolis, SC, v.13, n. 2, p. 759-783, abr./jun. 2013. 
fase à estratégia de manutenção preditiva e a gestão de boas práticas de manutenção, tal como ocorre em ambiente TPM. Entende-se como manutenção preditiva aquela baseada em medições feitas continuamente ou em intervalos periódicos, fornecendo a tendência evolutiva dos equipamentos. Caso algum defeito seja detectado, é feito o diagnóstico e analisada a tendência e uma intervenção de manutenção é executada antes da data prevista para a falha (MACCHI et al., 2012).

O último período é chamado por Mahmood et al. (2011) de desgaste e por Sellitto (2005) de mortalidade senil do equipamento. Nesta fase, ocorre aumento gradual na taxa de falhas, causado por envelhecimento, fadiga, degradação ou vida de projeto muito curta. Os autores apontam como adequada para esta fase a estratégia de manutenção preventiva. Nesta, o equipamento é substituído ou reformado. Geralmente, a decisão preventiva se vale de inspeções sistemáticas e da condição atual do equipamento. As tarefas são planejadas com base no tempo decorrido, no volume de produção acumulada, na quilometragem já percorrida ou no número de ciclos em máquinas rotativas (GARG e DESHMUKH, 2006). Na manutenção preventiva, há redução do risco de paradas não programadas, mas também pode haver intervenções desnecessárias, acarretando custo mais alto de operação (MACCHI et al., 2012; MAHMOOD et al., 2011). Uma programação de intervenções preventivas pode ser útil, podendo o tempo parado ser compartilhado com outras atividades fabris, tais como trocas de ferramentas e treinamento de operadores (LEÃO e SANTOS, 2009).

\section{A PESQUISA}

O objetivo deste artigo foi definir a estratégia de manutenção para uma bomba centrífuga de 600 HP instalada em uma planta petrolífera. O método de pesquisa foi a modelagem quantitativa. Modelos quantitativos usam linguagem matemática e técnicas analíticas ou estatísticas para descrever fenômenos e variáveis a estudar (MORABITO e PUREZA, 2010). A estratégia de manutenção a ser escolhida é uma entre as seguintes alternativas: emergencial, preventiva, preditiva ou corretiva. Para tanto, foi obtida a função risco da bomba e seu MTBF, segundo o modelo de Weibull. Com isto, foi possível situar a bomba na curva da banheira e escolher uma estratégia de manutenção. Adicionalmente, foi obtida a Revista Produção Online, Florianópolis, SC, v.13, n. 2, p. 759-783, abr./jun. 2013. 
função mantenabilidade e o MTTR da bomba, pela distribuição lognormal. Foi usado o software modelador ProConf 2000. De posse dos dois parâmetros de média, foi calculada a disponibilidade atual da bomba.

O Proconf 2000 é um programa computacional projetado para o ajuste de amostras de tempos de falha e de reparo a distribuições de probabilidade conhecidas: exponencial, Weibull, gamma, lognormal e normal. O software ajusta os dados por máxima verossimilhança e testa o ajuste pelos testes do qui-quadrado e de Kolmogorov-Smirnov. Para cada teste, o software informa o nível de significância. Vale o menor dos dois. Se for mais do que 5\%, a distribuição ajustada não pode ser rejeitada. Podem-se obter análises gráficas e matemáticas dos dados de falha. $O$ software oferece as funções de confiabilidade já mencionadas, seus parâmetros, parâmetros de tendência central e de dispersão e respectivos intervalos de confiança (FRITSCH e RIBEIRO, 1998).

Se mais do que uma distribuição não puder ser rejeitada, deve haver alguma fundamentação teórica que justifique a escolha por uma delas. Para o interesse deste artigo, a análise teórica aponta as distribuições de Weibull e lognormal como candidatas a modelarem os tempos entre falhas (TBF) e até o reparo (TTR). Assumiu-se antes da pesquisa que, se estas distribuições não fossem rejeitadas, as demais não interessariam.

A bomba centrífuga é um equipamento tecnológico composto por subsistemas, cujos modos de falha competem entre si pela falha geral: o primeiro componente ou subsistema que falhar causa a falha sistêmica. Esta estrutura de falha remete à distribuição de Weibull. Além disto, a parada desta bomba centrífuga indisponibiliza o posto de bombeio formado por mais uma bomba idêntica e outras duas de menor porte, caracterizando um sistema que opera em paralelo.

Os principais subsistemas que compõem esta bomba centrífuga são o selo mecânico, o eixo, os impelidores, os mancais de rolamentos, a motorização e sua alimentação elétrica, o elemento acoplador e a instrumentação de controle. A função deste equipamento é fornecer a vazão, requerida por meio de um contrato de abastecimento firmado com um cliente externo, de um produto petroquímico com características agressivas aos materiais, ao meio-ambiente e a saúde das pessoas.

O método de pesquisa foi a modelagem. O método de trabalho foi: 
- revisão bibliográfica dos conceitos de confiabilidade e mantenabilidade aplicados à manutenção de equipamentos industriais;

- delimitação do sistema a ser estudado;

- levantamento histórico de intervalos entre falhas e de tempos para reparo do objeto de estudo;

- modelagem, pela distribuição de Weibull, dos intervalos entre falhas e, identificação do ponto no ciclo de vida em que o equipamento se encontra, através do fator de forma obtido;

- modelagem, pela distribuição lognormal, dos tempos até o reparo;

- cálculo da disponibilidade do equipamento pelos valores esperados das funções de confiabilidade e mantenabilidade; e

- discussão dos resultados e proposta de uma política de manutenção para a bomba centrífuga.

\subsection{Modelagens dos TTR e TBF e disponibilidade}

A partir do sistema de informação da empresa, foram identificados e levantados dados de intervalos de tempo entre intervenções de manutenção de emergência entre os anos de 2001 e 2011. Foram consideradas apenas paradas não programadas do equipamento. Para cada intervenção de manutenção, existe um registro feito pela Engenharia de Manutenção da empresa, de onde foram coletados os dados para análise. O sistema de informações arredonda os dados para hora cheia, portanto não há sentido em apresentar fração de hora.

Os dados empregados são apresentados na Tabela 1.

Tabela 1 - Tempo até o reparo e tempo entre falhas.

\begin{tabular}{|c|c|c|c|c|c|c|c|c|c|}
\hline \multicolumn{10}{|c|}{ Tempos coletados } \\
\hline \multicolumn{5}{|c|}{ Tempo até o reparo (TTR, horas) } & \multicolumn{5}{|c|}{ Tempo entre falhas (TBF, horas) } \\
\hline 8 & 24 & 8 & 408 & 96 & 9336 & 1728 & 240 & 936 & 1224 \\
\hline 264 & 96 & 24 & 8 & 72 & 2544 & 2376 & 1224 & 72 & 12.912 \\
\hline 168 & 408 & 48 & 24 & 336 & 17.136 & 336 & 2976 & 5736 & 48 \\
\hline 72 & 192 & 8 & 24 & 120 & 888 & 168 & 6768 & 3720 & 576 \\
\hline 48 & 8 & 72 & 24 & 360 & 2088 & 144 & 7704 & 11.208 & \\
\hline
\end{tabular}

Fonte: Sistema de informação da empresa

Iniciou-se pela modelagem do tempo até o reparo.

Revista Produção Online, Florianópolis, SC, v.13, n. 2, p. 759-783, abr./jun. 2013. 
Pelo ProConf 2000, testou-se o ajuste pela lognormal. O ProConf 2000 não rejeitou a hipótese de que os dados se ajustem a esta distribuição. Portanto, pela premissa de pesquisa, as demais distribuições foram abandonadas. O teste de aderência é apresentado na Figura 2. A significância do ajuste é 0,178, o que é suficiente para o caso.

Figura 2 - Teste de aderência lognormal para o tempo até o reparo (Fonte: ProConf, 2000) TESTES DE ADERÊNCIA Teste do Qui-Quadrado: $\chi 2=1,42$ com 3 graus de liberdade $\quad$ Nível de Significância $=0,7011$

Teste de Kolmogorov-Smirnov:

DN $=0,1377 \quad$ Nível de Significância $=0,178$

A hipótese de que a população segue o modelo Lognormal não pode ser rejeitada.

Uma análise adicional que pode ser útil e que é oferecida pelo ProConf 2000 é o papel de probabilidade. Para a distribuição lognormal, o papel de probabilidade para os dados de TTR é apresentado na Figura 3.

Figura 3 - Papel de probabilidade lognormal para o tempo para reparo (Fonte: ProConf, 2000)

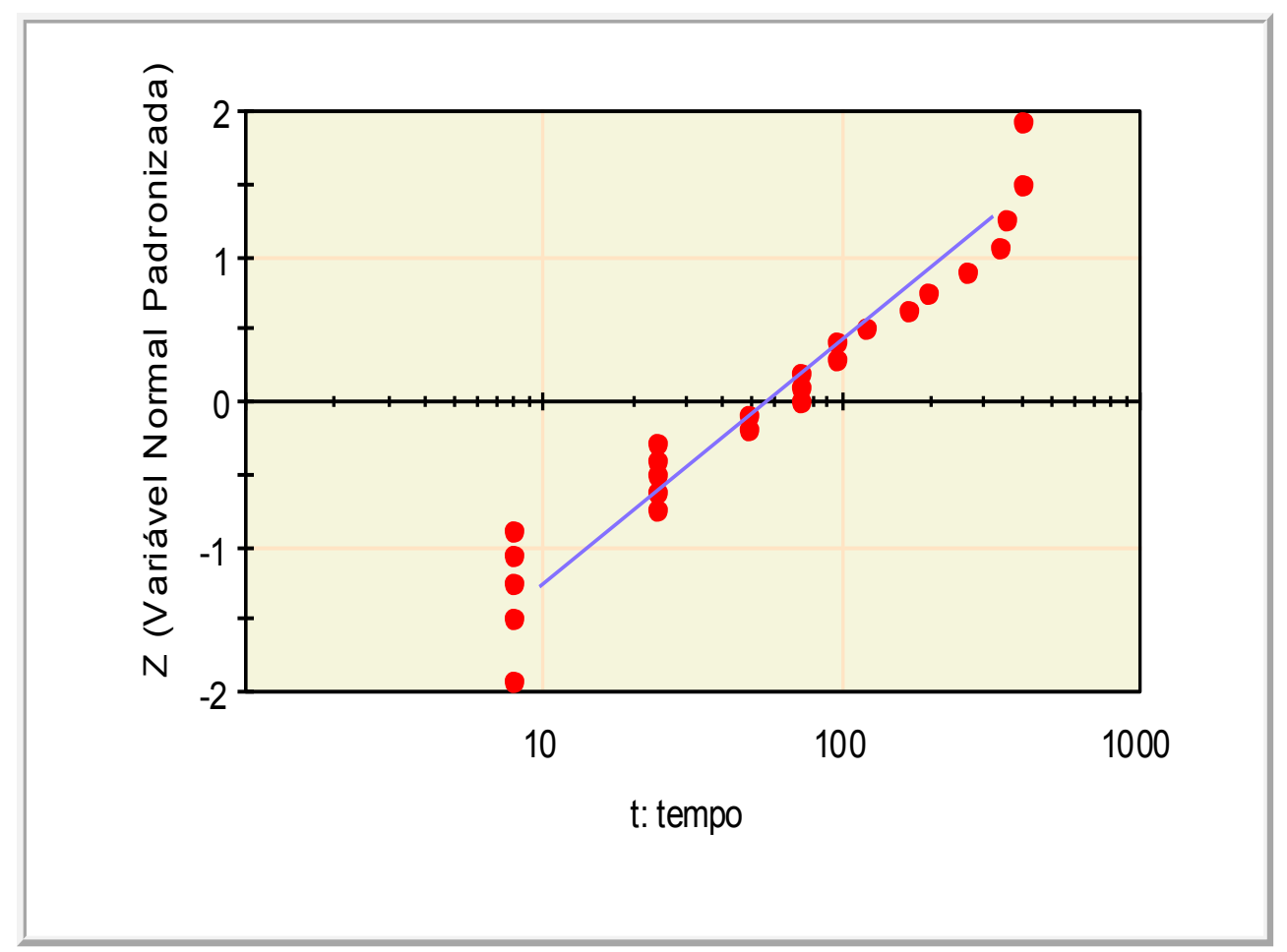


Na figura, observam-se acúmulos nas partes mais baixas. Caso o sistema de informação da empresa discriminasse frações de hora, talvez a curva fosse mais contínua nesta parte.

O tempo médio para reparo MTTR é de 133,02 horas e seu intervalo de confiança a $95 \%$ vai de 70,25 a 270,89 horas. O ajuste oferecido pelo ProConf 2000 é apresentado na Figura 4. A função mantenabilidade é representada pela Figura 5.

Figura 4 - Modelo lognormal para o tempo até o reparo (Fonte: ProConf, 2000)

\section{AJUSTE DOS DADOS - MODELO LOGNORMAL}

\section{Média do logarítmo dos dados $=4,0184$}

95\% do Intervalo de Confiança: para a média do logarítmo dos dados $=3,4736$ até 4,5631

Variância do logaritmo dos dados $=1,7444$

$95 \%$ do Intervalo de Confiança para a variância do logaritmo dos dados $=1,1076$ até 3,517

Tempo médio até a falha $=133,0294$

$95 \%$ do Intervalo de Confiança para o tempo médio até a falha $=70,2529$ até 270,8926

Var. do tempo médio até a falha $=83573,009$

$t_{10}=10,2319$

$t_{50}=55,6103$

Figura 5 - Função $M(t)$ mantenabilidade (Fonte: ProConf, 2000)

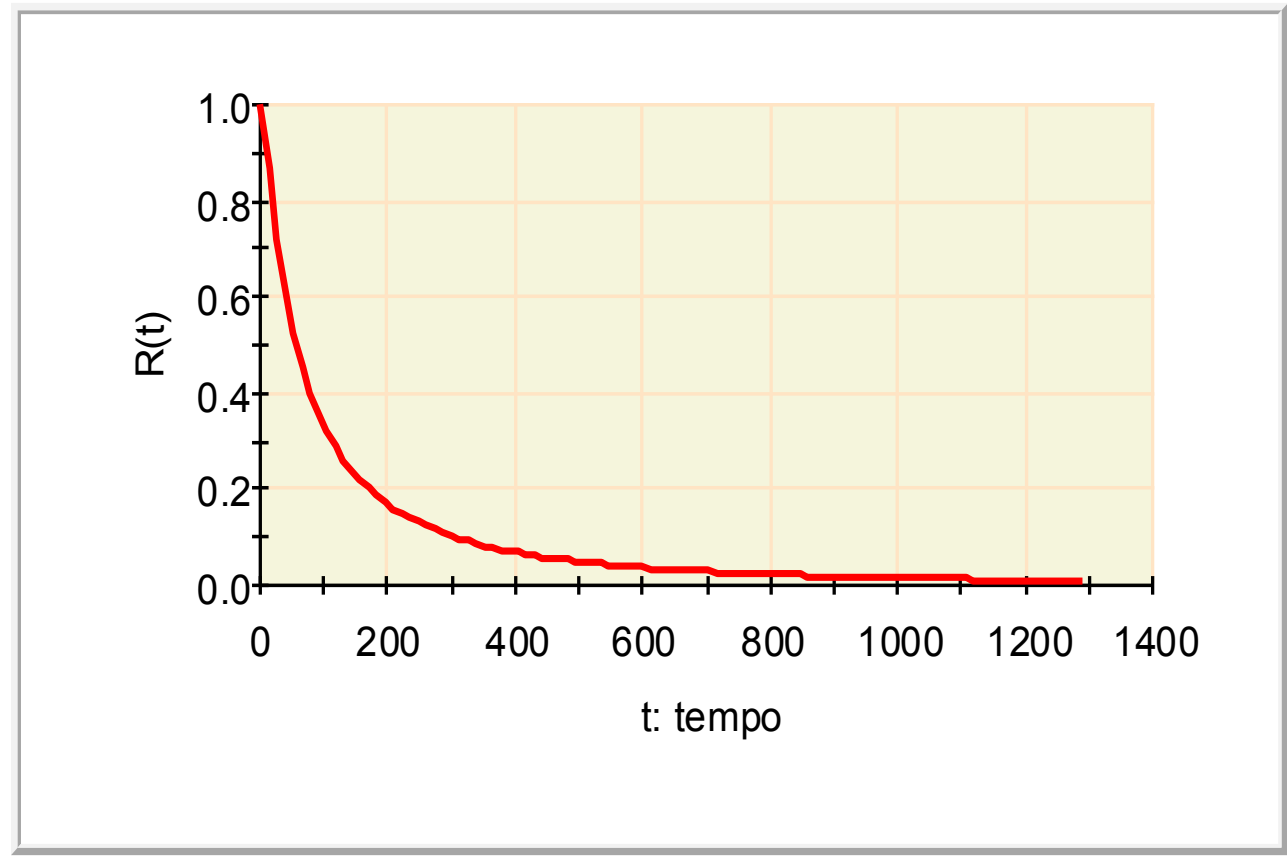

Revista Produção Online, Florianópolis, SC, v.13, n. 2, p. 759-783, abr./jun. 2013. 
Prosseguiu-se pela modelagem do tempo entre falhas.

Pelo ProConf 2000, testou-se o ajuste pelo distribuição de Weibull. O ProConf 2000 não rejeitou a hipótese de que os dados se ajustam a esta distribuição. Portanto, pela premissa de pesquisa, as demais distribuições foram abandonadas. $O$ teste de aderência é apresentado na Figura 6. A significância do ajuste é 0,391 , o que é suficiente para o caso.

Figura 6 - Teste de aderência Weibull para o tempo entre falhas (Fonte: ProConf, 2000)

TESTES DE ADERÊNCIA

Teste do Qui-Quadrado:

X2 $=1,65$ com 2 graus de liberdade Nível de Significância $=0,4387$

Teste de Kolmogorov-Smirnov:

DN $=0,077 \quad$ Nível de Significância $=0,391$

A hipótese de que a população segue o modelo Weibull não pode ser rejeitada.

O papel de probabilidade para o modelo Weibull é apresentado na Figura 7. A função confiabilidade da bomba centrífuga é apresentada na Figura 8.

Figura 7 - Papel de probabilidade Weibull para tempo entre falhas (Fonte: ProConf, 2000)

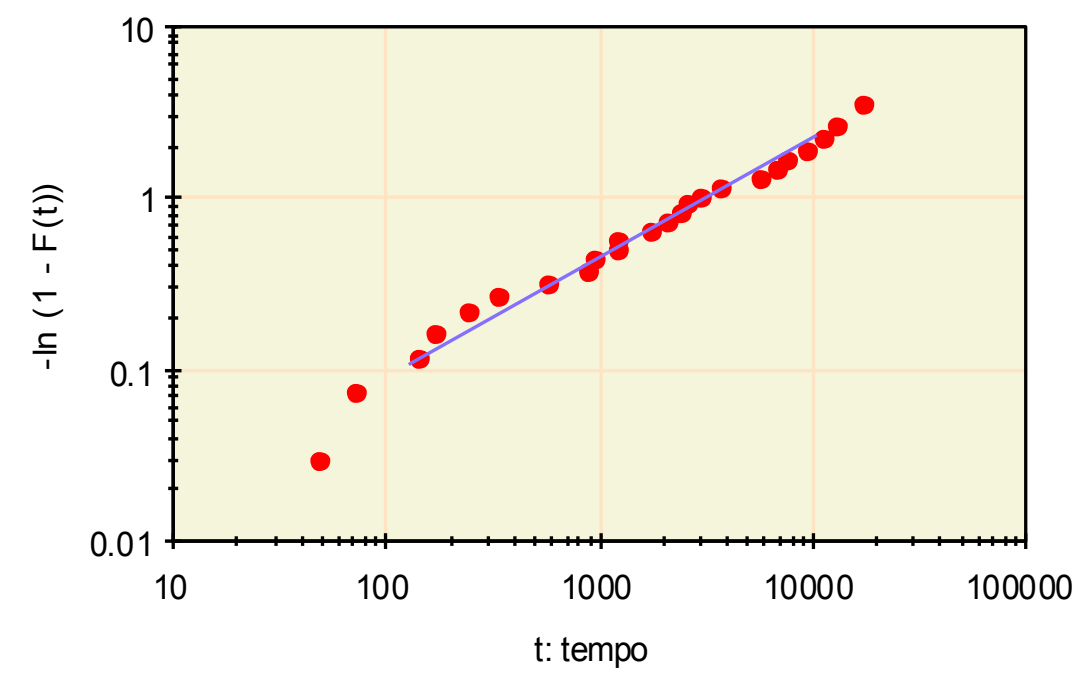

Revista Produção Online, Florianópolis, SC, v.13, n. 2, p. 759-783, abr./jun. 2013. 
Figura 8 - Função $R(t)$, confiabilidade (Fonte: ProConf, 2000)

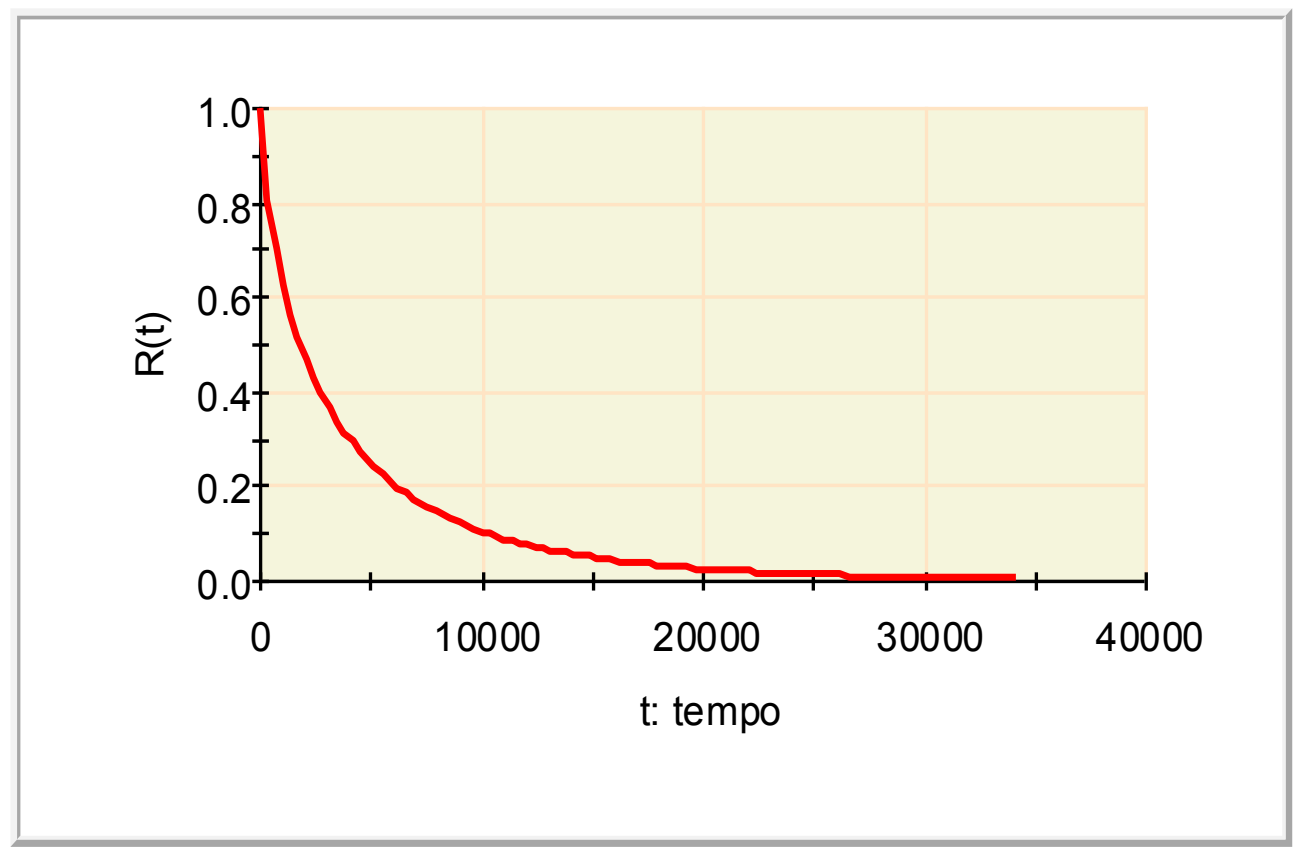

A modelagem para o tempo entre falhas pelo modelo Weibull é apresentada na Figura 9. Além da estimativa por máxima verossimilhança, o ProConf 2000 oferece uma estimativa não-tendenciosa, corrigida por algoritmo explicado em Sellitto et al. (2002). Para o interesse deste artigo, vale a estimativa não tendenciosa. Portanto, o fator de forma da distribuição assumida para explicar os TBF da bomba é 0,69 , o que indica mortalidade infantil e sugere escolher a estratégia de manutenção corretiva.

Figura 9 - Modelo Weibull para o tempo entre falhas (Fonte: ProConf, 2000)

\footnotetext{
AJUSTE DOS DADOS - MODELO WEIBULL

Parâmetro de Localização $=0$

Estimativa não tendenciosa:

Gamma $=0,6932$

$\operatorname{Var}($ Gamma $)=0,014$

Theta $=3080,429$

95\% do Intervalo de Confiança:

para Gamma $=0,4668$ até 0,8942

para Theta $=1536,611$ até 5808,69

$t_{10}=119,8708$

$t_{50}=1815,448$

MTTF $=3936,19$
} 
A função confiabilidade da bomba é dada pela equação (7), com $t$ em horas.

$$
R(t)=e^{-\left(\frac{t}{3080}\right)^{0,69}}
$$

O MTBF é de 3936 horas. Utilizando-se a equação (6), obtém-se pela equação (8) a disponibilidade do equipamento estudado. Pode-se então dizer que em $96,73 \%$ do tempo, a bomba centrífuga esteve disponível para a operação.

$$
A v(t)=\frac{3936}{3936+133}=96,73 \%
$$

\subsection{Estratégia de manutenção para o equipamento}

Pela análise de Weibull, obteve-se um fator de forma para a bomba de 0,69. Na Figura 10, apresenta-se a posição mais provável que o equipamento ocupa na curva da banheira. A estratégia indicada é a manutenção corretiva.

Figura 10 - Posição da bomba na curva da banheira

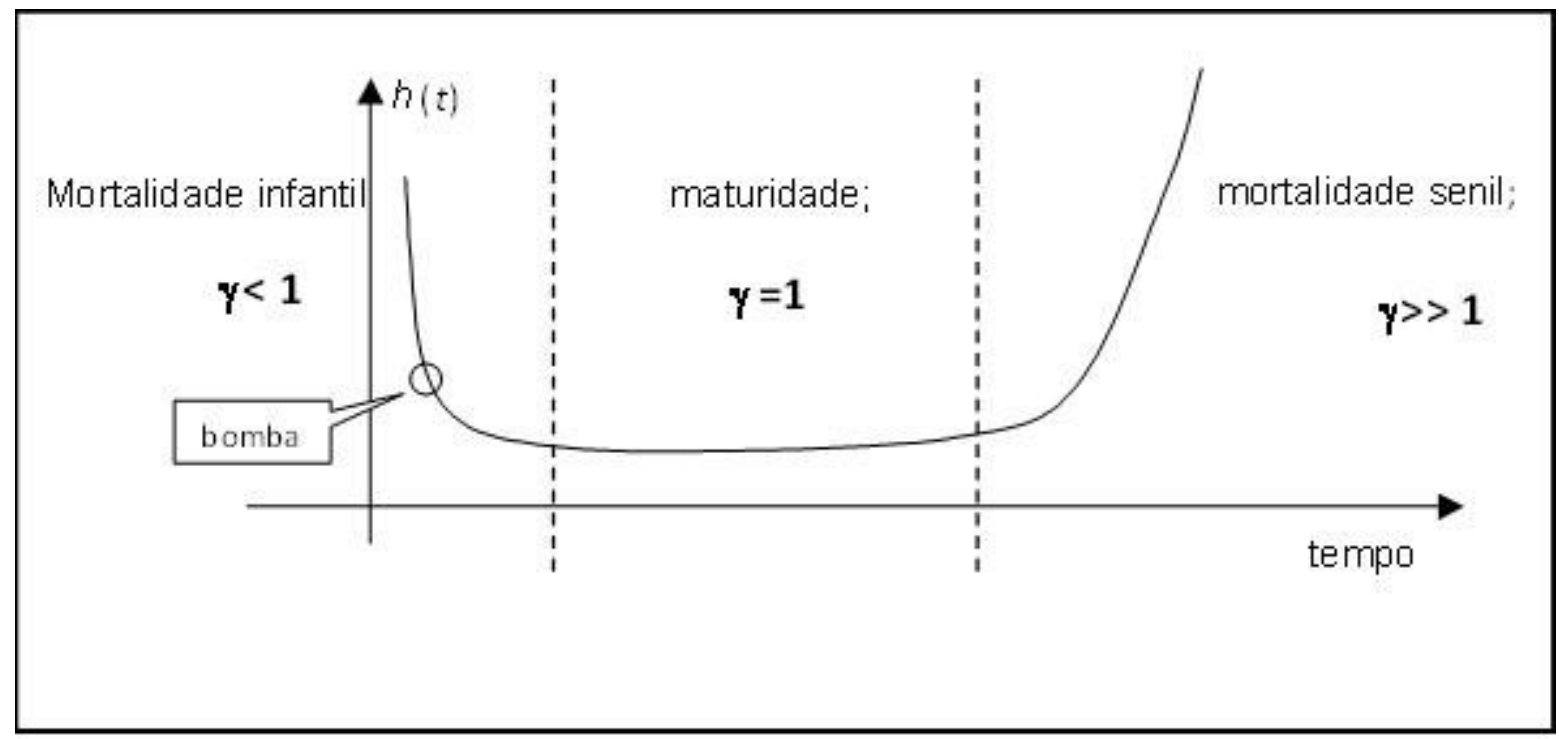

Revista Produção Online, Florianópolis, SC, v.13, n. 2, p. 759-783, abr./jun. 2013. 
A bomba centrífuga estudada neste artigo é atendida pela Engenharia de Manutenção e pelo setor de manutenção de equipamentos. A equipe usa uma estratégia de manutenção preditiva. Esta estratégia realiza a monitoração para predizer o momento em que se iniciará o processo de desgaste.

Após iniciada a operação do equipamento, a monitoração poderá predizer o momento em que aumenta a chance de quebra do mesmo, por meio de monitoração de variáveis-chave: vibração de rolamentos e análise de contaminação de óleo lubrificante. O objetivo do monitoramento é acompanhar falhas em progresso para evitar a possível quebra da bomba centrífuga. As principais falhas que têm sido observadas são apresentadas na Tabela 2. Observa-se que a principal falha apresentada na tabela, vazamento ou falha das selagens, é caracterizada pela impossibilidade de acompanhamento preditivo, pois as falhas possivelmente acontecem em virtude de deficiências estruturais do projeto.

Tabela 2 - Diagnóstico das falhas ao longo do tempo

\begin{aligned} & \hline Tipo de falhas Incidência das falhas \\ & \hline vazamento ou falha das selagens $55 \% \\ &$ problema na motorização e alimentação elétrica $14 \% \\ &$ problema de instrumentação $15 \% \\ &$ falha dos mancais $12 \% \\ &$ falha do elemento acoplador $4 \% \\ &$\hline\end{aligned}

Fonte: Sistema de informação da empresa

A falha de maior incidência, vazamento ou falha das selagens, apresentada pelos registros da empresa, pode ser explicado fato de tratar-se de um equipamento que sofreu alterações na configuração das selagens ao longo dos anos de operação. No projeto original da bomba centrífuga, a vedação do produto bombeado era feita por meio de anéis de gaxetas, que são elementos pré-formados e de estrutura mole. Os selos mecânicos de tecnologia superior, utilizados atualmente para esta finalidade, foram instalados em função da legislação que reduziu os limites de emissão de contaminantes para a atmosfera. Contudo, as características dos demais componentes da bomba centrífuga não foram estudadas durante esta modificação. Esta melhoria feita no equipamento necessita de soluções técnicas 
para uma operação confiável, como o aumento da rigidez do eixo para menor flexão e a alteração do tipo de elemento acoplador para menor vibração, por exemplo.

Como causas contribuintes para a redução da confiabilidade do posto de bombeio, têm-se as características negativas de operação das bombas centrífugas, como a aspiração difícil, necessidade de escorva e possibilidade de cavitação que causam danos severos às selagens, além das particularidades do fluído bombeado que podem potencializar estas características. Somado a isto, existem as especificidades de operação da bomba centrífuga, como procedimentos estabelecidos pelo fabricante do equipamento e das selagens, que são de responsabilidade do cliente, o que demanda o seu treinamento e comprometimento.

Como o equipamento se encontra na fase de mortalidade infantil, a Engenharia de Manutenção pode optar pela manutenção corretiva, conforme mostrado no Quadro 1, adaptado de Sellitto (2005). No quadro, o autor discorre sobre as consequências mais prováveis da adoção de quatro estratégias de manutenção em um equipamento que esteja em mortalidade infantil. Segundo o autor, a única estratégia que não traz algum tipo de prejuízo nesta fase é a manutenção corretiva, pois esta pode reduzir ou até eliminar as falhas prematuras, originadas principalmente de problemas de projeto ou de instalação.

Quadro 1 - Ciclo de vida e estratégia de manutenção

\begin{tabular}{|c|c|l|}
\hline Fase & Estratégia & \multicolumn{1}{c|}{ Resultados } \\
\hline $\begin{array}{c}\text { mortalidade } \\
\text { infantil }\end{array}$ & emergência & $\begin{array}{l}\text { retarda ou até impede o fim da mortalidade infantil ao não reforçar os } \\
\text { itens que quebraram ou não remover as causas das falhas de origem }\end{array}$ \\
\cline { 2 - 4 } & corretiva & $\begin{array}{l}\text { antecipa o fim da mortalidade infantil ao reforçar os itens que quebraram } \\
\text { ou remover as causas das falhas de origem }\end{array}$ \\
\cline { 2 - 3 } & preditiva & $\begin{array}{l}\text { monitora as falhas em progresso que podem resultar em quebra, mas } \\
\text { estas são muito poucas nesta fase, pois as quebras se dão mais por } \\
\text { baixa resistência }\end{array}$ \\
\cline { 2 - 3 } & preventiva & $\begin{array}{l}\text { perpetua ou até agrava a mortalidade infantil ao trocar exatamente os } \\
\text { sobreviventes, os itens fortes, que não têm falhas de origem }\end{array}$ \\
\hline
\end{tabular}

Fonte: Adaptado de Sellitto (2005)

Esta estratégia pode ser implementada em conjunto com representantes de fabricantes, pelo estudo técnico das principais falhas e visando sempre a reforçar os componentes por meio de alterações nos projetos originais da bomba centrífuga. $\mathrm{O}$ sistema de relatórios e histórico das intervenções permite o controle da eficácia destas ações, na medida em que permite que se acompanhe a evolução dos 
defeitos. A redução de sua incidência e a migração do equipamento ao longo da curva da banheira em direção à maturidade será uma indicação do sucesso das ações, que poderão ser revisadas e estendidas no tempo e a outros equipamentos.

O setor de Engenharia de Manutenção, responsável pela confiabilidade do equipamento foco deste estudo, possui um adequado sistema de informações, mantido por uma equipe de Tecnologia da Informação com qualificação material e humana adequada à necessidade da organização. Portanto, outra opção para tratar o equipamento em estudo e as demais bombas centrífugas de sua responsabilidade, é a utilização destes sistemas de informação visando o aprimoramento da gestão. Isto pode ser feito introduzindo-se técnicas quantitativas de confiabilidade nos moldes do que foi proposto nesta pesquisa.

Como se dispõem de informações consistentes sobre as intervenções de manutenção, é possível modelar os principais sistemas e subsistemas presentes na bomba centrífuga, descobrindo com isso as distribuições de probabilidade adequadas, segundo os modelos Weibull e lognormal, que explicam o seu comportamento, permitindo o planejamento de intervenções para que se mantenha, por exemplo, um determinado percentual de disponibilidade deste equipamento.

Por fim, existe a possibilidade de aumentar a disponibilidade do equipamento por redução do tempo de manutenção utilizando a Troca Rápida de Ferramenta (TRF) e o PDCA, conforme realizado por Leão e Santos (2009), permitindo sua máxima utilização em função do tempo para reparo. Porém, esta ação depende do planejamento da atividade de manutenção. De acordo com Santos (2006), outro modo de contribuir para o aumento da disponibilidade dos equipamentos é pela aplicação de um programa de controle e revisão dos fornecedores. Para isso, é necessário que se estabeleçam os requisitos relacionados com a mantenabilidade nos produtos e componentes entregues pelos fornecedores, visando este aumento de disponibilidade. Esta ação visa garantir que os itens pertencentes ao projeto e adquiridos dos fornecedores sejam compatíveis com os requisitos do próprio programa de mantenabilidade da empresa. 


\section{CONSIDERAÇÕES FINAIS}

O objetivo deste artigo foi definir a estratégia de manutenção adequada para uma bomba centrífuga instalada em uma planta petrolífera (escolhida entre preventiva, preditiva, corretiva ou emergencial), baseada em cálculos de confiabilidade. Isto foi possível com a definição da posição no ciclo de vida e na curva da banheira deste sistema tecnológico complexo, sujeito ao desgaste e a intervenções incompletas de manutenção. O método de pesquisa foi a modelagem.

Os dados foram originados do histórico gerenciado pela Engenharia de Manutenção da empresa estudada. Nota-se neste momento a importância do acompanhamento registrado e da qualidade destas informações para o desenvolvimento deste tipo de atividade por parte dos gestores da manutenção de equipamentos, possibilitando inferir e decidir corretamente, além de adquirir os modelos que melhor se enquadram ao sistema estudado.

O estudo tem valor para a empresa, pois esta bomba centrífuga pertence a um posto de bombeio, que além dela possui outra bomba idêntica e duas de menor porte. Este posto fica indisponível com a parada de qualquer uma das quatro bombas, ou seja, caracteriza um sistema que opera em paralelo. No caso de falha deste sistema, é acionando um outro posto de capacidade reduzida, o qual fica operando até que o sistema seja reestabelecido.

Além disto, a orientação do setor de Engenharia de Manutenção pelos princípios da RCM, a qual se baseia em um modelo teórico, nos moldes do que foi proposto neste artigo, possibilita determinar datas prováveis da ocorrência das falhas ou a curva de perda de confiabilidade da operação dos equipamentos. $O$ indicador disponibilidade pode aumentar a competitividade da empresa, pois quanto maior a disponibilidade dos equipamentos importantes, maiores os lotes e menores os custos unitários de produção.

O método contribuiu para verificar que a estratégia manutenção preditiva, utilizada neste equipamento pela empresa, não está adequada para a sua posição na curva da banheira. A mortalidade infantil apontada no resultado sugere a manutenção corretiva, a qual buscaria as reais causas dos possíveis defeitos de fabricação ou de projeto e as eliminaria. Adotando-se esta estratégia, é necessário que na ocorrência de uma falha, a substituição do componente que falhou seja feita 
por um item inspecionado. O erro de especificação, projeto ou fabricação deve ser reconhecido e ao menos bloqueado, se não for possível eliminá-lo. A consequência de não se fazer a manutenção corretiva é a impossibilidade de se entrar na região de maturidade ou vida útil do equipamento.

\section{REFERÊNCIAS}

BRAND, G. Método de apoio à formulação de estratégia de manutenção em ambiente industrial: um estudo de caso. Dissertação de Mestrado, Universidade do Vale do Rio dos Sinos, Engenharia de Produção e Sistemas, São Leopoldo: 2002.

DODSON, B.; NOLAN, D. Reliability engineering handbook. New York: Marcel Dekker, 2002.

ELSAYED, E. System reliability engineering. Reading, Massachusetts: Addison Wesley Longman, 1996.

FOGLIATTO, F.; RIBEIRO, J. Confiabilidade e manutenção industrial. Rio de Janeiro: Elsevier, 2009.

FRITSCH, C.; RIBEIRO, J. ProConf: um software orientado para análises de confiabilidade. In: XVIII ENCONTRO NACIONAL DE ENGENHARIA DE PRODUÇÃO, 1998. Anais... Rio de Janeiro: 1998.

GARG, A; DESHMUKH, S. Maintenance management: literature review and directions. Journal of Quality in Maintenance Engineering, v.12, n.3, p.205-238, 2006.

GROSH, D. A primer of reliability theory. New York: John Wiley \& Sons, 1989.

GUZZON, S. Proposta de análise quantitativa de confiabilidade a partir de dados qualitativos provenientes da FMEA. Dissertação (Mestrado em Engenharia de Produção) - Universidade Federal do Rio Grande do Sul. Porto Alegre, 2009.

HAHN, G.; SHAPIRO, S. Statistical models in engineering. New York: John Wiley \& Sons, 1967.

IRESON, W.; COOMBS, C.; MOSS, R. Handbook of reliability engineering and management. New York: McGray-Hill, 1996.

KAPUR, K.; LAMBERSON, L. Reliability in engineering design. New York: John Wiley \& Sons, 1977.

LAFRAIA, J. Manual de confiabilidade, mantenabilidade e disponibilidade. Rio de Janeiro: Qualitymark: Petrobrás, 2001. 
LEÃO, S.; SANTOS, M. Aplicação da troca rápida de ferramentas (TRF) em intervenções de manutenção preventiva. Produção Online, Santa Catarina, v.9, n. 1, p.01-026, 2009.

LEWIS, E. Introduction to reliability engineering. 2. ed. New York: John Wiley \& Sons, 1996.

LOPES, L.; SAMOHYL, R. Análise de componentes principais: técnica alternativa de análise de confiabilidade em sistemas complexos multivariados. Revista Produção Online, Santa Catarina, v.3, n.2, p.01-14, 2003.

LUCATELLI, M. Proposta de aplicação da manutenção centrada em confiabilidade em equipamentos médico-hospitalares. Dissertação (Mestrado)Universidade Federal de Santa Catarina, Engenharia de Produção e Sistemas. Florianópolis, 2002.

MACCHI, M.; GARETTI, M.; CENTRONE, D.; FUMAGALLI, L.; PAVIRANI, G. Maintenance management of railway infrastructures based on reliability analysis. Reliability Engineering \& Systems Safety, v.104, n.1, p.71-83, 2012.

MAHMOOD, W.; RAHMAN, M.; DEROS, B.; MAZLI, H. Maintenance management system for upstream operations in oil and gas industry: a case study. International Journal of Industrial and Systems Engineering, v.9, n.3, p.317-329, 2011.

MÁRQUEZ, A.; GUPTA, J. Contemporary maintenance management: process, framework and supporting pillars. Omega, v.34, n.2, p.313-326, 2006.

MÁRQUEZ, A.; LÉON, P.; FERNÁNDES, J.; MÁRQUEZ, C.; CAMPOS, M. The maintenance management framework: a practical view to maintenance management. Journal of Quality in Maintenance Engineering, v.15, n.2, p.167-178, 2009.

MENDES, A. Manutenção Centrada em Confiabilidade: uma abordagem quantitativa. 2011. 85 f. Dissertação (Mestrado em Engenharia de Produção) Programa de Pós-Graduação em Engenharia de Produção, Universidade Federal do Rio Grande do Sul. Porto Alegre, RS, 2011.

MORABITO, R.; PUREZA, V. Modelagem e simulação. In: MIGUEL, P. (org.) Metodologia de pesquisa em engenharia de produção e gestão de operações. Rio de Janeiro: Campus, 2010.

NELSON, W. Applied Life Data Analysis. New York: John Wiley \& Sons, 1982.

NIKOLOPOULOS, K.; METAXIOTIS, K.; LEKATIS, N.; ASSIMAKOLOULOS, V. Integrating industrial maintenance strategy into ERP. Industrial Management and Data Systems, v.103, n.3, p.184-191, 2003.

PARDIA, A.; CHATTOPADHYAY, G. Development of a multi-criteria hierarchical framework for maintenance performance measurement (MPM). Journal of Quality in Maintenance Engineering, v.13, n.3, p.241-258, 2007. 
PINJALA, S.; PINTELON, L.; VERECKA, A. An empirical investigation on the relationship between business and maintenance strategies. International Journal of Production Economics, v.104, n.3, p.214-229, 2006.

PROCONF2000. Confiabilidade de componentes: software ProConf 2000. Porto Alegre: Maxxi Gestão Empresarial, 2000.

RAMOS FILHO, J.; ATAMANCZUK, M.; MARÇAL, R. Seleção de técnicas de manutenção para processo de armazenagem pelo método de análise hierárquica. Produção Online, v.10, n.1, p.142-166, 2010.

RAPOSO, C. Overall Equipment Effectiveness: aplicação em uma empresa do setor de bebidas do polo industrial de Manaus. Produção Online, v.11, n.3, p.648-667, 2010.

RAPOSO, J. Manutenção centrada em confiabilidade aplicada a sistemas elétricos: uma proposta para uso de analise de risco no diagrama de decisão. Dissertação (Mestrado em Engenharia Elétrica) - Universidade Federal da Bahia. Salvador, 2004.

RAUSAND, M.; HOYLAND, A. System reliability theory. N.York: Wiley Interscience, 2004.

SALGADO, M. Aplicação de técnicas de otimização a engenharia de confiabilidade. Dissertação (Mestrado em Engenharia Elétrica) - Universidade Federal de Minas Gerais. Belo Horizonte, 2008.

SANTOS, I. Metodologia para implementação de um programa de manutenibilidade no ciclo de vida de um sistema nuclear. Produção Online, v.6, n.3, p.1-28, 2006.

SANTOS, W. Determinação da periodicidade da manutenção preventiva em sistemas reparáveis. Dissertação (Mestrado)-Departamento de Estatística, UFMG. Belo Horizonte, 2003.

SELLITTO, M. Análise estratégica da manutenção de uma linha de fabricação metalmecânica baseada em cálculos de confiabilidade de equipamentos, GEPROS, v.2, n.1, p.97-108, 2007.

SELLITTO, M. Formulação estratégica da manutenção industrial com base na confiabilidade dos equipamentos. Produção, v.15, n. 01, p.044-059, 2005.

SIQUEIRA, I. Manutenção centrada na confiabilidade: manual de implementação. Rio de Janeiro: Qualitymark, 2009.

TSANG, A. Strategic dimensions of maintenance management. Journal of Quality in Maintenance Engineering, v.8, n.1, p.7-39, 2002. 
WUTTKE, R.; SELLITTO, M. Cálculo da disponibilidade e da posição na curva da banheira de uma válvula de processo petroquímico. Produção Online, v.8, n.4, p.123, 2008.

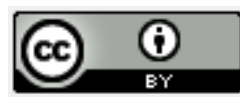

Artigo recebido em 13/06/2012 e aceito para publicação em 16/12/2012. 